\title{
Assessment of Pelvic Floor Function and Quality of Life in Patients Treated for Cervical Cancer: A Multicenter Retrospective Study
}

\author{
Sha Wang ${ }^{a, b, c}$ Hongwu Wen ${ }^{d}$ Yunong Gao ${ }^{e}$ Qiubo Lv ${ }^{f}$ Tingting Cao $^{a, b, c}$ \\ Shiyan Wanga,b,c Jing Wang ${ }^{a}$ Yi Li $^{g}$ Haibo Wangh ${ }^{\text {Zhiqi Wang }}{ }^{\text {a,b, c }}$ \\ Xiuli Sun ${ }^{a, b, c}$ Jianliu Wang ${ }^{a, b, c}$

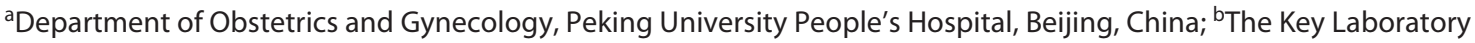 \\ of Female Pelvic Floor Disorders Disease, Beijing, China; 'The Research Center of Female Pelvic Floor Disorders \\ Disease of Peking University, Beijing, China; ${ }^{\mathrm{d} D e p a r t m e n t}$ of Obstetrics and Gynecology, Peking University First \\ Hospital, Beijing, China; 'Department of Gynecology, Peking University Cancer Hospital and Institute, Beijing, China; \\ fDepartment of Obstetrics and Gynecology, Beijing Hospital, Beijing, China; ${ }^{9}$ Peking University Medical Information

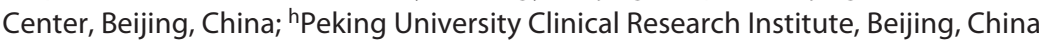

\section{Keywords \\ Cervical cancer - Radical hysterectomy - Radiotherapy · \\ Pelvic floor function - Lower urinary tract symptoms . \\ Defecation symptoms · Quality of life}

\begin{abstract}
Objectives: Our study aimed to evaluate the quality of life (QoL) and pelvic floor function of cervical cancer (CC) patients after treatment. Design: This was a cross-sectional observational cohort study. Participants: The participants included in this study were CC patients who underwent radical hysterectomy (RH) from 2012 to 2018 at 18 medical centers across China. Methods: The validated versions of the Pelvic floor Distress Inventory-Short Form 20, Overactive Bladder Symptom Score, and Euro Qol Five-Dimension questionnaires were used to evaluate postoperative pelvic floor dysfunction (PFD) and QoL. Results: A total of 689 CC patients were enrolled. The incidence of stress urinary incontinence (SUI), incomplete urinary emptying, and constipation were $32.7,27.7$, and $28.6 \%$, respectively. Multivariate analysis confirmed that laparoscopic $\mathrm{RH}(\mathrm{LRH})$ and vaginal wall resection greater than $3 \mathrm{~cm}$ were risk factors for lower urinary tract
\end{abstract}

karger@karger.com www.karger.com/goi

Karger"

BOPEN ACCESS
(C) 2021 The Author(s)

Published by S. Karger AG, Basel

This is an Open Access article licensed under the Creative Commons Attribution-NonCommercial-4.0 International License (CC BY-NC) (http://www.karger.com/Services/OpenAccessLicense), applicable to the online version of the article only. Usage and distribution for commercial purposes requires written permission. symptoms (LUTS). LRH and chemotherapy were risk factors for SUl. Chemoradiotherapy and LRH were risk factors for overactive bladder (OAB). A high body mass index and $L R H$ were risk factors for more severe defecation symptoms. ARH and large amount of operative blood loss were risk factors for poor QoL. Conclusion: PFD is common in CC patients after treatment. LRH seems to increase the postoperative distress, including LUTS and defecation symptoms. Postoperative urinary incontinence and $O A B$ are more bothersome for patients undergoing chemotherapy and radiotherapy. We recommend evaluating pelvic floor function as a standard assessment during follow-up. @ 2021 The Author(s)

Published by S. Karger AG, Basel

\section{Introduction}

Cervical cancer (CC) is the fourth most common cancer in women worldwide. In developing countries, this disease is the leading cause of cancer-related death in women and also represents a major public health problem [1]. Women with CC presenting with International Federation of Gynecology and Obstetrics (FIGO) stage
Correspondence to:

Xiuli Sun, sunxiuli918@126.com

Jianliu Wang,wangjianliu1203@163.com 
IA, IB, and small IIA tumors are preferably treated with radical hysterectomy $(\mathrm{RH})$ and pelvic lymphadenectomy, with or without adjuvant chemoradiotherapy, depending on the pathology results [2]. However, the complications of treatment have persistent negative effects on the physical and psychosocial function of patients. $\mathrm{RH}$ involves more extensive removal of the vagina, uterosacral ligaments, and parametrial tissue. Damage to the autonomic nerve tissue is likely to occur during $\mathrm{RH}$ and cause pelvic floor disorders [3]. Pelvic floor dysfunction (PFD) is widely recognized as a public health issue with significant impact on quality of life (QoL) for millions of women globally [4]. Radical surgical treatment is especially known to affect bladder function, resulting in lower urinary tract symptoms (LUTS), with an incidence varying from 12 to $85 \%$ due to the use of different definitions and diagnostic tools [5]. Radiotherapy of the pelvis is also known to cause adverse effects on bladder and bowel function, such as overactive bladder (OAB) symptoms and anal incontinence [6]. Unfortunately, few studies have examined postoperative pelvic floor function and QoL in CC patients. This study aimed to evaluate QoL and pelvic organ function for CC patients after treatment and identify the risk factors of distress.

\section{Materials and Methods}

This is a multicenter cross-sectional observational cohort study. The study protocol was approved by the Institutional Review Boards of Peking University People's Hospital, and the study is registered at www.clinicaltrials.gov (NCT02492542). We recruited CC patients who underwent RH from June 2012 to June 2018 at 18 medical centers across China. The inclusion criteria were as follows: (1) patients aged over 18 years; (2) patients with a diagnosis of CC who underwent $\mathrm{RH}$; (3) patients with a follow-up duration of 3-24 months after surgery; and (4) patients who agreed to participate in the study and signed the informed consent form. The exclusion criteria were as follows: previous urinary and bowel symptoms, anti-incontinence procedure, or pelvic surgery. Selected patients were invited to the hospitals to sign the informed consent form and were asked to answer specific questionnaires. The validated versions of the Pelvic Floor Distress Inventory-Short Form 20 (PFDI-20), Overactive Bladder Symptom Score (OABss), and Euro Qol Five-Dimension (EQ-5D) questionnaires were used in a detailed evaluation of postoperative PFD and QoL. The PFDI20 was divided into the Urogenital Distress Inventory (UDI-6) and the Colorectal Anal Distress Inventory (CRADI-8). The official Chinese versions of the questionnaires were used. LUTS, OAB, and defecation were evaluated by the validated versions of the UDI-6, OABss, and CRADI-8. Higher scores on these questionnaires indicated worsening of symptoms. The UDI- 6 can be divided into several subscales, and stress urinary incontinence (SUI) was evaluated by questions 3 and 4 . The EQ-5D questionnaire is designed to be self-administered by patients. The questionnaire includes 5 functional domains (mobility, self-care, usual activities, pain/discomfort, and anxiety/depression) and the EQ visual analog scale (EQ VAS). The EQ VAS records the patient's self-rated health using a vertical VAS, where the end points are labeled "The best health you can imagine" and "The worst health you can imagine." It is used to measure the health outcome that reflects the patient's own judgment. Based on the questionnaire scores, we investigated the postoperative pelvic floor function and health status of patients and analyzed the related factors.

All patients underwent type III $\mathrm{RH}$, with or without nervesparing techniques. The operations were performed by skilled surgeons with extensive training and experience in gynecologic oncology, using standardized surgical approaches. In addition, some patients received adjuvant therapy according to the clinical stage and pathology results. Specimens of the length of resected tissues were measured after surgery by the surgeon.

All demographic, surgical, and oncological data were calculated and reported. Demographic variables included age $(<45$ or $\geq 45$ years), body mass index $\left(\mathrm{BMI} ; \mathrm{kg} / \mathrm{m}^{2}\right)$, parity $(<2$ or $\geq 2)$, and medical history of increased abdominal pressure (yes or no). Diseaserelated variables included the FIGO stage of disease (categorized as [1] IA; [2] IB1+IIA1; [3] IB2+IIA2; or [4] IIB), adjuvant chemoradiotherapy (yes vs. no), pathological type, and time interval between date of treatment and date of completion of the questionnaire. Surgical variables included the operation time (hours), operative blood loss, operation approach (laparoscopic vs. abdominal), nerve-sparing procedure (yes vs. no), and the length of resected vaginal wall and ligament.

SPSS program version 24.0 was used for statistical analyses. The statistical methods employed included Pearson's $\chi^{2}$ test and a generalized estimating equation. The generalized estimating equation was performed to identify variables that were significantly associated with postoperative pelvic floor function and QoL. $p=0.05$ was accepted as the degree of significance. Data are given as the mean \pm standard deviation or percentage.

\section{Results}

A total of 718 women met the criteria and were originally recruited in this study. However, 29 patients were excluded because of loss of data. Therefore, 689 patients were considered for the final analysis. The demographic and disease-related characteristics of the patients are shown in Table 1. Patient ages ranged from 23 to 78 years, and $66.5 \%$ of the patients were older than 45 years. The average BMI was $23.91 \mathrm{~kg} / \mathrm{m}^{2}$, and approximately $11.0 \%$ of the patients were obese. According to the FIGO stage, $5.1,73.3,16.7$, and $4.9 \%$ of the patients were classified as IA, IB1+IIA1, IB2+IIA2, and IIB, with most presenting in the early stage. Based on the preference and experience of surgeons, $60.4 \%$ of the patients underwent laparoscopic RH (LRH), and 39.6\% underwent abdominal RH (ARH). The follow-up time ranged from 3 to 24 months, and the median time was 12 months. 
Table 1. Demographic characteristics and disease-related data of patients

\begin{tabular}{|c|c|}
\hline Characteristics & $N(\%)$ \\
\hline \multicolumn{2}{|l|}{ Age } \\
\hline$<45$ years & $231(33.5)$ \\
\hline$\geq 45$ years & $458(66.5)$ \\
\hline $\mathrm{BMI}$ & $23.91 \pm 3.16$ \\
\hline$<18.5$ & $15(2.2)$ \\
\hline $18.5-23.9$ & $355(51.5)$ \\
\hline $24.0-27.9$ & $243(35.3)$ \\
\hline$\geq 28.0$ & $76(11.0)$ \\
\hline \multicolumn{2}{|l|}{ Parity } \\
\hline$<2$ & $382(55.4)$ \\
\hline$\geq 2$ & $307(44.6)$ \\
\hline \multicolumn{2}{|l|}{ FIGO stage } \\
\hline IA & $35(5.1)$ \\
\hline IB1+IIA1 & $505(73.3)$ \\
\hline IB2+IIA2 & $115(16.7)$ \\
\hline IIB & $34(4.9)$ \\
\hline \multicolumn{2}{|l|}{ Pathological type } \\
\hline Squamous cell carcinoma & $557(80.8)$ \\
\hline Adenocarcinoma & $96(14.0)$ \\
\hline Adenosquamous carcinoma & $12(1.7)$ \\
\hline Others & $24(3.5)$ \\
\hline \multicolumn{2}{|l|}{ Operation approach } \\
\hline Laparoscopic & $416(60.4)$ \\
\hline Abdominal & $273(39.6)$ \\
\hline \multicolumn{2}{|l|}{ Treatment modalities } \\
\hline Surgery only & $220(31.9)$ \\
\hline Surgery + chemotherapy & $176(25.5)$ \\
\hline Surgery + chemoradiotherapy & $293(42.5)$ \\
\hline \multicolumn{2}{|l|}{ Follow-up time } \\
\hline 3-6 months & $158(22.9)$ \\
\hline $6-12$ months & $203(29.5)$ \\
\hline $12-24$ months & $328(47.6)$ \\
\hline
\end{tabular}

FIGO, International Federation of Gynecology and Obstetrics; BMI, body mass index.

Table 2 displays the self-reported prevalence of pelvic floor symptoms after treatment. CC patients had a significantly increased risk of urinary dysfunction. The results showed that $32.7 \%$ of patients complained about SUI, which was the most common symptom among patients in this study, based on the responses to the specific question of the UDI-6. In addition, $27.7 \%$ of patients reported incomplete emptying of urine, $28.6 \%$ reported constipation, and $24.4 \%$ reported incomplete emptying of stool.

Table 3 shows the results of the univariate and multivariate logistic regression analysis for the factors related to postoperative pelvic floor function and QoL, re-
Table 2. The incidence of postoperative urinary and defecation symptoms

\begin{tabular}{lc}
\hline Symptoms & Incidence (\%) \\
\hline Urinary function & \\
Urge UI & $121(17.6)$ \\
SUI & $225(32.7)$ \\
Incomplete emptying of urine & $191(27.7)$ \\
Frequent urination & $96(13.9)$ \\
Defecation function & \\
Constipation & $197(28.6)$ \\
Incomplete emptying of stool & $168(24.4)$ \\
Fecal incontinence & $41(6.0)$ \\
Painful defecation & $60(8.7)$
\end{tabular}

SUI, stress urinary incontinence; UI, urinary incontinence.

spectively. The univariate analysis indicated that a low BMI, surgical LRH, length of the resected uterosacral ligament $\leq 3 \mathrm{~cm}$, and low operative blood loss were significantly protective factors for QoL according to the EQ-5D score. Age over 45 years old, LRH, length of the resected cardinal ligament, uterosacral ligament or vaginal wall greater than $3 \mathrm{~cm}$, and operation time longer than 4 hours were significant risk factors for LUTS based on the UDI-6 score. Chemotherapy and LRH were significant risk factors for SUI. Parity $\geq 2$, chemoradiotherapy, LRH, length of the resected cardinal ligament, uterosacral ligament or vaginal wall greater than $3 \mathrm{~cm}$, and follow-up time were significantly related to the OABss. Increased BMI, parity $\geq 2, \mathrm{LRH}$, and length of the resected cardinal ligament greater than $3 \mathrm{~cm}$ were significantly related to severe defecation distress according to the CRADI- 8 score.

The multivariate analysis results are shown in Table 4. In the multivariate analysis, a high BMI and large amount of operative blood loss were independent risk factors for poor QoL (odds ratio $[\mathrm{OR}]=1.330,95 \%$ confidence interval [CI]: $1.007-1.758$, OR $=1.376,95 \% \mathrm{CI}: 1.170$ 1.830 , respectively), and LRH was an independent protective factor $(\mathrm{OR}=0.099,95 \% \mathrm{CI}: 0.013-0.723)$. LRH was an independent risk factor for LUTS $(\mathrm{OR}=1.167,95 \% \mathrm{CI}$ : $1.068-1.276$ ), and a vaginal wall resection length $\leq 3 \mathrm{~cm}$ was an independent protective factor based on the UDI-6 score (OR $=0.867,95 \%$ CI: $-0.740-0.982)$. LRH and chemotherapy were independent risk factors for SUI (OR = 1.495, 95\% CI: $1.064-2.099$, OR $=2.357$, 95\% CI: $1.111-$ 4.998 , respectively). Parity $\leq 2$ was a protective factor for $\mathrm{OAB}(\mathrm{OR}=0.565,95 \%$ CI: 0.390-0.819), while chemo- 
Table 3. Univariate analysis of the related factor to pelvic floor function and QoL

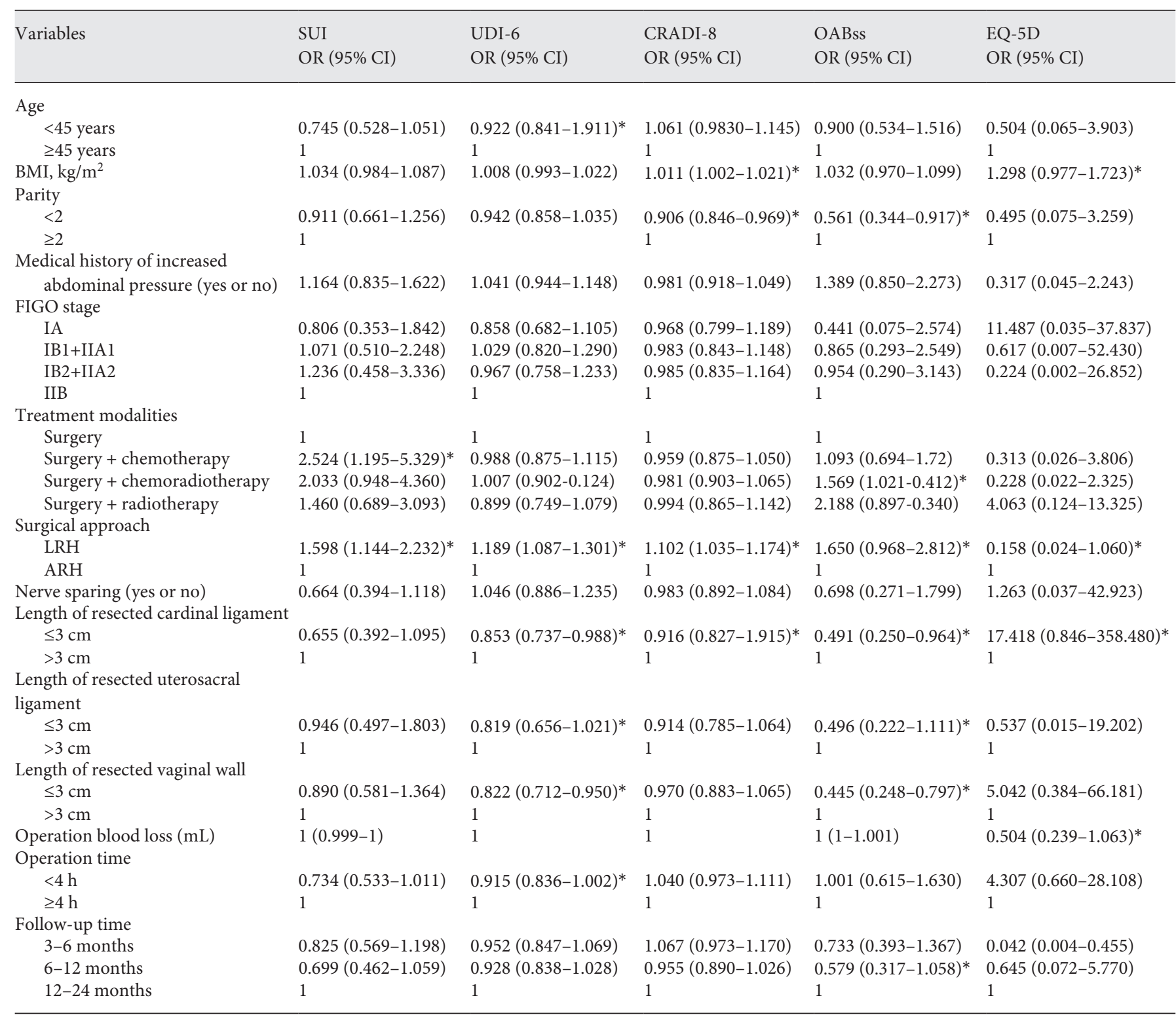

BMI, body mass index $\left(\mathrm{kg} / \mathrm{m}^{2}\right)$; FIGO, International Federation of Gynecology and Obstetrics; LRH, laparoscopic radical hysterectomy; ARH, abdominal radical hysterectomy; OABss, Overactive Bladder Symptom Score; SUI, stress urinary incontinence; UDI-6, Urogenital Distress Inventory; CRADI-8, Colorectal Anal Distress Inventory; EQ-5D, Euro Qol Five-Dimension; QoL, quality of life; OR, odds ratio; CI, confidence interval. Data presented as OR ( $95 \%$ CI) unless otherwise specified. $* p<0.05$ indicates statistical significance.

radiotherapy, and $\mathrm{LRH}$ were risk factors for $\mathrm{OAB}(\mathrm{OR}=$ 1.549, 95\% CI: $1.002-2.396$, OR $=1.592$, 95\% CI: $1.112-$ 2.280, respectively). A high BMI and LRH were independent risk factors for worse defecation symptoms $(\mathrm{OR}=$ $1.011,95 \%$ CI: $1.002-1.021$, OR $=1.100$, 95\% CI: $1.032-$ 1.171 , respectively), and parity $\leq 2$ was a protective factor $(\mathrm{OR}=0.906,95 \%$ CI: 0.847-0.969).
Table 5 shows the results of the operation-related variables of patients in the LRH and ARH groups. Statistically significant differences were observed in terms of length of the resected cardinal ligament and vaginal wall ( $p=0.002, p<0.001$, respectively), which indicated that LRH results in a larger range of parametrial tissue resection. 
Table 4. Multivariate analysis of the related factor to pelvic floor function and QoL

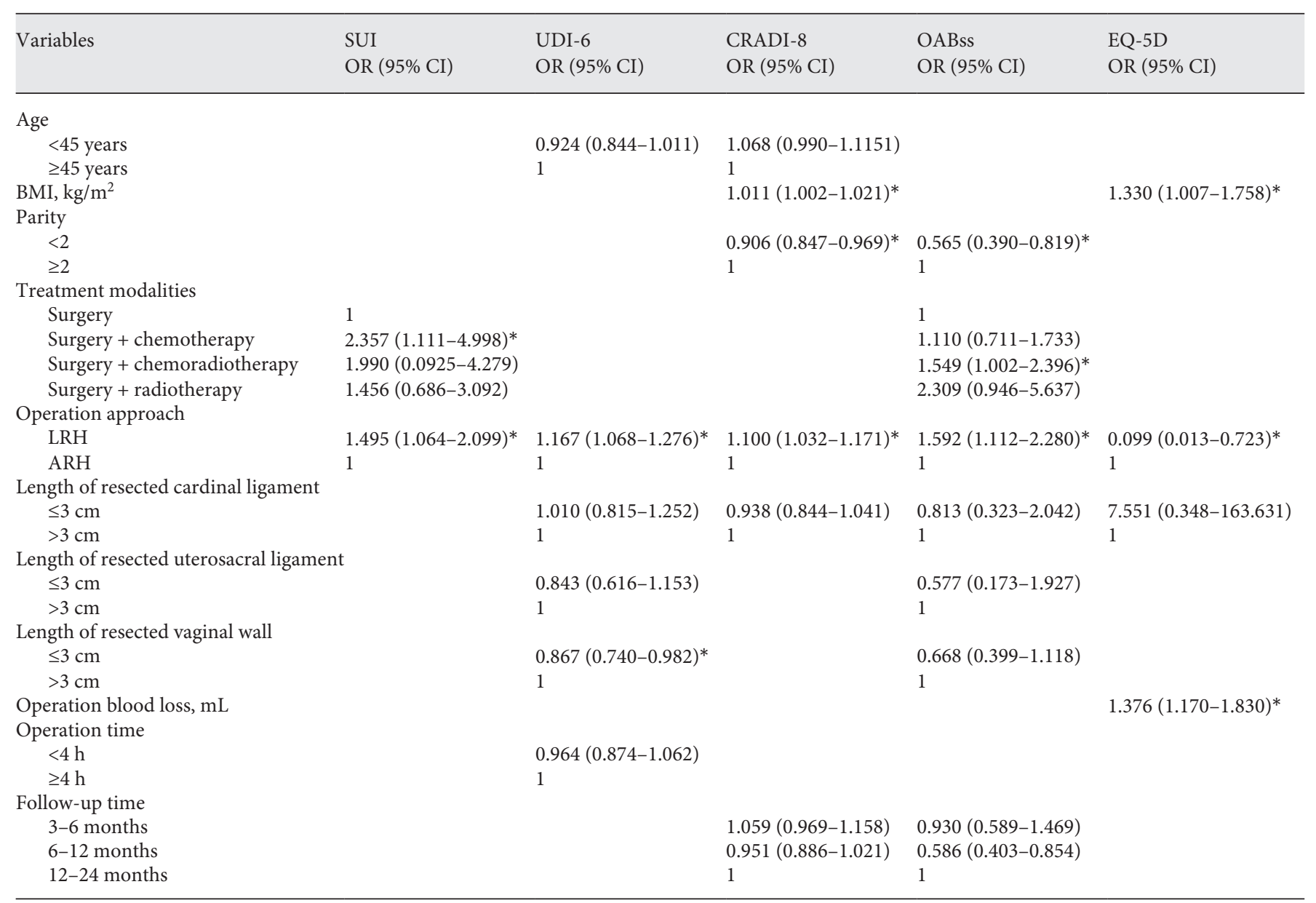

BMI, body mass index $\left(\mathrm{kg} / \mathrm{m}^{2}\right)$; LRH, laparoscopic radical hysterectomy; ARH, abdominal radical hysterectomy; OABss, Overactive Bladder Symptom Score; SUI, stress urinary incontinence; UDI-6, Urogenital Distress Inventory; CRADI-8, Colorectal Anal Distress Inventory; EQ-5D, Euro Qol Five-Dimension; QoL, quality of life; OR, odds ratio; CI, confidence interval. Data presented as OR (95\% CI) unless otherwise specified. ${ }^{*} p<0.05$ indicates statistical significance.

\section{Discussion}

CC is one of the most widespread gynecological malignancies in women worldwide. In China, CC is associated with a high incidence and mortality rate, which severely damages the physical and mental health of patients [7]. Currently, RH with pelvic lymphadenectomy is the standard treatment for early stage CC patients. This treatment regimen has a 5-year overall rate of up to $80 \%$ [8]. However, RH is associated with long-term adverse effects on pelvic floor function. Many patients complain of pelvic floor symptoms such as UI, OAB, constipation, and sexual dysfunction after surgery [9]. Moreover, some studies have reported that surgery with adjuvant chemoradio- therapy is associated with urological complications that are twice as severe and gastrointestinal adverse events that are 3 times more severe than those reported after surgery alone [10]. In recent years, oncologists have focused on the overall survival of CC patients. However, QoL and pelvic floor function are also important but often ignored factors.

The results of our study after a long-term follow-up demonstrate that CC patients have bothersome pelvic floor symptoms postoperatively. UI is the most pronounced symptom. The incidence of SUI is $32.7 \%$ and that of incomplete urinary emptying is $27.7 \%$. Constipation is the most often reported colorectal symptom after $\mathrm{RH}$, with a prevalence of $28.6 \%$. Other studies have re- 
Table 5. The operation-related variables of the patients between LRH and ARH group

\begin{tabular}{lllc}
\hline & $\begin{array}{l}\text { LRH } \\
(n=416), n(\%)\end{array}$ & $\begin{array}{l}\text { ARH } \\
(n=273), n(\%)\end{array}$ & $p$ value \\
\hline $\begin{array}{l}\text { Length of resected cardinal ligament }>3 \mathrm{~cm} \\
\text { Length } \text { of resected uterosacral ligament }>3 \mathrm{~cm}\end{array}$ & $53(12.7)$ & $15(5.5)$ & $0.002^{*}$ \\
Length of resected vaginal wall $>3 \mathrm{~cm}$ & $30(7.2)$ & $14(5.1)$ & 0.274 \\
$<0.001^{*}$
\end{tabular}

LRH, laparoscopic radical hysterectomy; ARH, abdominal radical hysterectomy. ${ }^{*} p<0.05$ indicates statistical significance.

ported that the incidence of postoperative urinary dysfunction varies from 12 to $85 \%$ [5]. The prevalence of intestinal dysfunction can reach $75 \%$ [11]. In addition, the difference in incidence is likely due to the diagnostic method of urinary symptoms, variation in the follow-up period, and variation in surgical techniques.

The advantages of laparoscopy in oncological surgery, such as minimal intraoperative blood loss, reduced postoperative pain, shorter hospitalization times, and reduction of health-care costs, have been described [12]. However, the difference in postoperative pelvic floor function between the laparoscopic and abdominal approach is still controversial. In our study, we found that LRH was a risk factor for LUTS, especially for postoperative symptoms of SUI and OAB. Postoperative LUTS occur due to partial or complete disruption of the sympathetic and parasympathetic fibers that pass through the paracervix and innervate the urethrovesical complex during resection of the uterosacral and rectovaginal ligaments, the dorsal and lateral paracervix, the caudal part of the vesicouterine ligaments, or the vagina [13]. We then compared the resection range of parametrial tissue between the LRH and ARH groups and found that LRH involved a longer resection length of the ligament and vaginal wall. Since the scope of laparoscopy is clearer than that of abdominal surgery, surgeons tended to perform longer resections to achieve better oncological outcomes. Therefore, we speculate that the larger ligament resection could explain the increased possibility of nerve injury with the laparoscopic approach compared with ARH and could result in long-term complications.

Although LRH may increase the prevalence of LUTS and defecation distress, we found that laparoscopic surgery resulted in a better physical and mental condition. Compared to ARH, laparoscopic surgery provides a clearer surgical field, which can avoid damage to the abdominal tissue or vascular structures and decrease the amount of blood loss during the operation. Moreover, the surgical procedure is mainly performed by electrocoagulation, which can also minimize the amount of blood loss. In addition, postoperative intestinal function recovers more quickly after laparoscopic than abdominal surgery, and the postoperative abdominal incision heals faster because of the minimal invasiveness. Obesity was a risk factor for poor QoL, and moderate exercise is helpful for obtaining a favorable physical condition as well as a pleasant mood. The results of previous studies showed that spiritual well-being and social support were predictive factors that significantly affected QoL among CC patients, and a better understanding of long-term QoL in CC survivors is crucial $[14,15]$.

Defecation symptoms are also bothersome for patients. Our results showed that a high BMI, parity $>2$, and laparoscopic surgery increased the severity of bowel symptoms. RH involves extensive dissection of the pelvic structures, which can cause increased damage to the nerves innervating of rectum. Hazewinkel et al. [10] reported that a great disturbance in body image was associated with severe defecation symptoms, which is similar to our results. Our findings are also consistent with those from Laterza et al. [16], who found that laparoscopic surgery increased the occurrence of obstructed defecation compared to abdominal surgery. Direct injury to the parasympathetic nerves lateral to the vaginal fornix and in the broad ligament of the uterus is responsible for posthysterectomy constipation [17].

Chemoradiotherapy is associated with greater distress caused by pelvic floor symptoms than in patients treated with $\mathrm{RH}$. The incidence of UI was $36 \%$ in patients who underwent RH with chemotherapy or radiotherapy [18]. In our study, the ORs of SUI and OAB were 2.4 and 1.549 , respectively, for patients who received chemotherapy and chemoradiotherapy compared to those with $\mathrm{RH}$ alone. Other studies showed similar results. Noronha et al. [19] 
reported that radiotherapy and chemoradiotherapy treatments for CC are more frequently associated with urinary and intestinal dysfunctions, with a higher incidence of urinary frequency and diarrhea, than surgery alone. Chemotherapy and radiotherapy increased the likelihood of some types of serious acute toxicity, particularly hematologic and gastrointestinal toxicity. However, acute toxicity and diarrhea during radiation therapy for CC significantly increase the risk of severe late toxicity and affect the patient's QoL. Radiotherapy is known to cause fibrosis in bladder walls, which results in a low compliance of the bladder and explains the elevated risk of urinary urgency and frequency in patients who undergo primary radiotherapy $[20,21]$. Therefore, oncologists should consider a treatment strategy to minimize the development of PFD following adjuvant therapy.

Currently, nerve-sparing $\mathrm{RH}$ has been introduced with the aim of reducing pelvic dysfunction related to conventional $\mathrm{RH}$. Because of the close proximity of the paracervix and supporting tissues around the uterus with the pelvic nerves, the integrity of pelvic innervation may be compromised during RH [22]. Although no significant differences between patients who underwent nerve-sparing procedures and those who underwent non-nerve-sparing procedures were observed in our study, some studies have demonstrated that nerve-sparing techniques appear to improve bladder function without compromising overall survival [23]. Ditto et al. [24] reported a significant difference between patients undergoing nerve-sparing $\mathrm{RH}$ and patients undergoing non-nerve-sparing $\mathrm{RH}$. The nerve-sparing group had better bladder function and a lower postoperative complication rate than the nerve-resection group. Another study found that $\mathrm{RH}$ with a non-nerve-sparing procedure, adjuvant radiotherapy, and voiding with abdominal pressure at 3 months after surgery were risk factors for persistent low bladder compliance [25]. The nervesparing approach during $\mathrm{RH}$ has become popular in recent years and can better identify visceral nerve fibers and surgical landmarks, thereby improving dissection of the vascular structures from the nerve tissue of the parametrium [26].

The following limitations of our study merit attention. First, the study was retrospective nature, and although the patients with previous urinary and bowel symptoms were excluded, we lacked information about preoperative pelvic floor function for comparison purposes. The second limitation is that we evaluated postoperative urinary and bowel function based on answers to questionnaires, which are subjective and can be influ- enced by the status or mood of patients. Therefore, the results might not accurately represent pelvic floor function. Objective evaluation and diagnostic methods should be used to detect pelvic nerve and muscle dysfunction. Additionally, the surgeries were completed by different oncological surgeons, and some subjective bias was present regarding the operation, which might be difficult to control.

A strength of our study was that we used validated selfreport questionnaires to assess a wide spectrum of pelvic floor symptoms. Additionally, we achieved a questionnaire response rate of $96 \%$. Our results showed that CC survivors have a high prevalence of urinary and bowel symptoms after treatment, and compared with abdominal surgery, laparoscopic surgery caused more distress. Furthermore, our results suggest that adjuvant therapy increased the adverse effects on pelvic floor function compared to RH alone. However, additional randomized controlled trials are needed to confirm these results.

\section{Conclusion}

PFD is common in CC patients who undergo $\mathrm{RH}$ with or without adjuvant therapy. Many patients complain about bothersome urinary and defecation dysfunction symptoms, especially patients who are obese, have high parity, and undergo laparoscopic surgery. The distress and symptoms considerably influence the patients' daily lives. These results emphasize the necessity to evaluate CC patients after treatment. Furthermore, evaluation of pelvic floor function, using methods such as a validated questionnaire, and objective examinations should be completed during the follow-up period. Performance of these assessments during follow-up visits could assist surgeons in considering treatments for bothersome symptoms, which will likely relieve distress and enhance the patient's QoL.

\section{Acknowledgements}

We gratefully acknowledge all of the patients who participated in our study and the assistance and support from medical workers in different medical centers.

\section{Statement of Ethics}

This study was approved by the Ethics Committee of Peking University People's Hospital, and the study is registered at www. clinicaltrials.gov (NCT02492542). 


\section{Conflict of Interest Statement}

None of the authors of this article have any conflict of interest, financial, or otherwise.

\section{Funding Sources}

This research was funded by the Beijing Municipal Science \& Technology Commission (CN) (Grant No. D151100001915003).

\section{Author Contributions}

Sha Wang contributed to manuscript writing, data collection, and data analysis; Hongwu Wen, Yunong Gao, and Qiubo Lv contributed to design of the study and data collection; Tingting Cao and Shiyan Wang contributed to data collection; Jing Wang, Yi Li, and Haibo Wang contributed to data analysis and interpretation of data; Zhiqi Wang contributed to design of the study; Xiuli Sun and Jianliu Wang contributed to design of the study and manuscript revision. All the authors approved the final version of the manuscript.

\section{References}

1 Arbyn M, Weiderpass E, Bruni L, Sanjosé S, Saraiya M, Ferlay J, et al. Estimates of incidence and mortality of cervical cancer in 2018: a worldwide analysis. Lancet Glob Health. 2020;8(2):e191-203.

2 Abu-Rustum NR, Yashar CM, Bean S, Bradley K, Campos SM, Chon HS, et al. NCCN guidelines insights: cervical cancer, version 1.2020. J Natl Compr Canc Netw. 2020;18(6): 660-6.

3 Thomas SG, Sato HRN, Glantz JC, Doyle PJ, Buchsbaum GM. Prevalence of symptomatic pelvic floor disorders among gynecologic oncology patients. Obstet Gynecol. 2013;122(5): 976-80.

4 Schofield C, Newton RU, Cohen PA, Galvão DA, McVeigh JA, Mohan GR, et al. Healthrelated quality of life and pelvic floor dysfunction in advanced-stage ovarian cancer survivors: associations with objective activity behaviors and physiological characteristics. Support Care Cancer. 2018;26(7):2239-46.

5 Aoun F, Roumeguère T. [Lower urinary tract dysfunction following radical hysterectomy]. Prog Urol. 2015;25(17):1184-90.

6 Takekuma M, Kasamatsu Y, Kado N, Kuji S, Tanaka A, Takahashi N, et al. The issues regarding postoperative adjuvant therapy and prognostic risk factors for patients with stage I-II cervical cancer: a review. J Obstet Gynaecol Res. 2017;43(4):617-26.

7 Colombo N, Carinelli S, Colombo A, Marini C, Rollo D, Sessa C, et al. Cervical cancer: ESMO Clinical Practice Guidelines for diagnosis, treatment and follow-up. Ann Oncol. 2012;23(Suppl 7):vii27-32.

8 Derks M, van der Velden J, Frijstein MM, Vermeer WM, Stiggelbout AM, Roovers JP, et al. Long-term pelvic floor function and quality of life after radical surgery for cervical cancer: a multicenter comparison between different techniques for radical hysterectomy with pelvic lymphadenectomy. Int J Gynecol Cancer. 2016;26(8):1538-43.

9 Axelsen SM, Petersen LK. Urogynaecological dysfunction after radical hysterectomy. Eur J Surg Oncol. 2006;32(4):445-9.
10 Hazewinkel MH, Sprangers MAG, Velden JVD, van der Vaart CH, Stalpers LJ, Burger $\mathrm{MP}$, et al. Long-term cervical cancer survivors suffer from pelvic floor symptoms: a crosssectional matched cohort study. Gynecol Oncol. 2010;117(2):281-6.

11 Plotti F, Angioli R, Zullo MA, Sansone M, Altavilla T, Antonelli E, et al. Update on urodynamic bladder dysfunctions after radical hysterectomy for cervical cancer. Crit Rev Oncol Hematol. 2011;80(2):323-9.

12 Corrado G, Vizza E, Legge F, Pedone Anchora L, Sperduti I, Fagotti A, et al. Comparison of different surgical approaches for Stage IB1 cervical cancer patients: a multi-institution study and a review of the literature. Int J Gynecol Cancer. 2018;28(5):1020-8.

13 Selcuk S, Cam C, Asoglu MR, Kucukbas M, Arinkan A, Cikman MS, et al. Effect of simple and radical hysterectomy on quality of life analysis of all aspects of pelvic floor dysfunction. Eur J Obstet Gynecol Reprod Biol. 2016; 198:84-8.

14 Khalil J, Bellefqih S, Sahli N, Afif M, Elkacemi $\mathrm{H}$, Elmajjaoui S, et al. Impact of cervical cancer on quality of life: beyond the short term (Results from a single institution). Gynecol Oncol Res Pract. 2015;2:7.

15 Greenwald HP, Mccorkle R, Baumgartner K, Gotay C, Neale AV. Quality of life and disparities among long-term cervical cancer survivors. J Cancer Surviv. 2014;8(3):419-26.

16 Laterza RM, Sievert KD, De Ridder D, Vierhout ME, Haab F, Cardozo L, et al. Bladder function after radical hysterectomy for cervical cancer. Neurourol Urodyn. 2015;34(4): 309-15.

17 Pieterse QD, Kenter GG, Maas CP, de Kroon CD, Creutzberg CL, Trimbos JB, et al. Selfreported sexual, bowel and bladder function in cervical cancer patients following different treatment modalities: longitudinal prospective cohort study. Int J Gynecol Cancer. 2013; 23(9):1717-25.

18 Roh JW, Lee DO, Suh DH, de Kroon CD, Creutzberg CL, Trimbos JB, et al. Efficacy and oncologic safety of nerve-sparing radical hysterectomy for cervical cancer: a randomized controlled trial. J Gynecol Oncol. 2015;26:909.
19 Noronha AF, Mello de Figueiredo E, Rossi de Figueiredo Franco TM, et al. Treatments for invasive carcinoma of the cervix: what are their impacts on the pelvic floor functions? Int Braz J Urol. 2013;39(1):46-54.

20 Benedetti-Panici P, Zullo MA, Plotti F, Manci N, Muzii L, Angioli R. Long-term bladder function in patients with locally advanced cervical carcinoma treated with neoadjuvant chemotherapy and type 3-4 radical hysterectomy. Cancer. 2004;100(10):2110-7.

21 Miguel TP, Laurienzo CE, Faria EF, Sarri AJ, Castro IQ, Júnior RJA, et al. Chemoradiation for cervical cancer treatment portends high risk of pelvic floor dysfunction. PLoS One. 2020;15(6):e0234389.

22 Balaya V, Guimiot F, Uhl JF, Ngo C, Delomenie $\mathrm{M}$, Bonsang-Kitzis $\mathrm{H}$, et al. Three-dimensional modelization of the female human inferior hypogastric plexus: implications for nerve-sparing radical hysterectomy. Gynecol Obstet Invest. 2019;84(2):196-203.

23 Lee YH, Kim MK, Moon HY, Chong GO, Lee HJ, Lee YS, et al. Proteomic analysis of pelvic autonomic nerve in nerve-sparing radical hysterectomy for cervical carcinoma. Cancer Genomics Proteomics. 2018 Jul-Aug; 15(4): 337-42.

24 Ditto A, Martinelli F, Mattana F, Reato C, Solima E, Carcangiu M, et al. Class III nervesparing radical hysterectomy versus standard class III radical hysterectomy: an observational study. Ann Surg Oncol. 2011;18(12):346978.

25 Oda Y, Todo Y, Hanley S, Hosaka M, Takeda $\mathrm{M}$, Watari $\mathrm{H}$, et al. Risk factors for persistent low bladder compliance after radical hysterectomy. Int J Gynecol Cancer. 2011;21(1): $167-72$.

26 Kruppa J, Kavvadias T, Amann S, Baessler K, Schuessler B, et al. Short and long-term urodynamic and quality of life assessment after nerve sparing radical hysterectomy: a prospective pilot study. Eur J Obstet Gynecol Reprod Biol. 2016;201:131-4. 